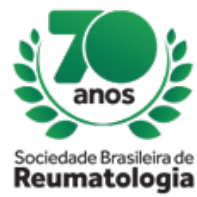

\title{
T-CELL LARGE GRANULAR LYMPHOCYTE LEUKEMIA, A RARE MANIFESTATION OF RHEUMATOID ARTHRITIS: CASE REPORT
}

Priscila Garcia Câmara Cabral Tavares (Hospital Universitário Walter Cantídio, Universidade Federal do Ceará, Fortaleza, CE, Brasil), Ana Paula de Menezes Cunha (Hospital Universitário Walter Cantídio, Universidade Federal do Ceará, Fortaleza, CE, Brasil), Ruy Sampaio Siqueira Neto (Hospital Universitário Walter Cantídio, Universidade Federal do Ceará, Fortaleza, CE, Brasil), André de Gois Rocha (Hospital Universitário Walter Cantídio, Universidade Federal do Ceará, Fortaleza, CE, Brasil), Leila Patrícia Fonseca Oliveira (Hospital Universitário Walter Cantídio, Universidade Federal do Ceará, Fortaleza, CE, Brasil), Cristiano Nogueira Marques (Hospital Universitário Walter Cantídio, Universidade Federal do Ceará, Fortaleza, CE, Brasil)

\section{BACKGROUND}

Rheumatoid arthritis (RA) is an inflammatory, systemic, autoimmune and chronic disease that affect joints with a destructive potential. It can also affect other organs and systems such as skin, heart, muscles, vessels. A variant of Felty's syndrome has been reported in patients with RA with neutropenia and increased numbers of large granular lymphocytes in blood and bone marrow.

\section{CASE REPORT}

Patient, 57 years old, female, from Fortaleza-CE, with rheumatoid arthritis since 1996. Over the years, has evolved with deformities despite optimal use of disease-modifying anti-rheumatic drugs (DMARDs) and corticosteroids. Periodic examinations showed normal levels of blood cells, however, in May, 2016 was detected significant and persistent leucopenia and neutropenia, in addition to recurrent infections. The medications in use were suspended due to the suspicion of myelotoxicity and a hematological investigation was started. On October 31, 2018, after myelogram, bone marrow biopsy, immunohistochemistry and karyotype banding, was diagnosed with large granular T-cell lymphocytic leukemia, CD8 positive, ruling out myelodysplastic syndromes and drug myelotoxicity. Methotrexate $(25 \mathrm{mg} /$ day) was started in November 2018 and suspended in early June 2019 due lack of response. It has been decided to treat with rituximab, however is awaiting a urinary infection resolution to begin the use.

\section{CONCLUSION}

T-Cell Large Granular Lymphocyte (LGL) Leukemia is a rare clonal disorder in which the main clinical manifestations are cytopenias (mainly neutropenia), splenomegaly, and recurrent infections. T cell LGL leukemia can be associated with RA, usually in patients with long-term disease, severe joint damage, and extra-articular manifestations. The diagnosis is based on the discovery of a monoclonal LGG expansion in the peripheral blood and also in the bone marrow with a characteristic immunophenotype (CD3+, CD4-, CD8+, CD16+, CD28- and CD57+). Generally, LGL leukemia has a chronic and indolent course, with an average survival of 10 years. The objectives of LGL leukemia therapy are the normalization of cytopenias and reduction of infectious episodes and also reduce the tumor burden to treat the underlying disease. As in RA, the treatment of choice is with methotrexate at the usual doses, with remission up to $50 \%$ of the cases; Alternatives are cyclosporin A, cyclophosphamide and rituximab. 